\title{
Article
}

\section{Effect of metal chelates on the ignition and early flaming behaviour of intumescent fire-retarded polyethylene systems}

WANG, D, LIU, Y, GE, X, WANG, Y, Stec, Anna A, BISWAS, B, Hull, T Richard and PRICE, D

Available at http://clok.uclan.ac.uk/1092/

WANG, D, LIU, Y, GE, X, WANG, Y, Stec, Anna A ORCID: 0000-0002-6861-0468, BISWAS, B, Hull, T Richard ORCID: 0000-0002-7970-4208 and PRICE, D (2008) Effect of metal chelates on the ignition and early flaming behaviour of intumescent fire-retarded polyethylene systems. Polymer Degradation and Stability, 93 (5). pp. 1024-1030. ISSN 01413910

It is advisable to refer to the publisher's version if you intend to cite from the work. http://dx.doi.org/10.1016/j.polymdegradstab.2007.12.011

For more information about UCLan's research in this area go to http://www.uclan.ac.uk/researchgroups/ and search for < name of research Group>.

For information about Research generally at UCLan please go to http://www.uclan.ac.uk/research/

All outputs in CLoK are protected by Intellectual Property Rights law, including Copyright law. Copyright, IPR and Moral Rights for the works on this site are retained by the individual authors and/or other copyright owners. Terms and conditions for use of this material are defined in the policies page. 
Polymer Degradation and Stability 93, 1024-1030, (2008).

\title{
Effect of metal chelates on the ignition and early flaming behaviour of intumescent fire-retarded polyethylene systems
}

\author{
De-Yi Wang ${ }^{\mathrm{a}, \mathrm{b}}$, Yun Liu ${ }^{\mathrm{a}}$, Xin-Guo Ge ${ }^{\mathrm{a}}$, Yu-Zhong Wang ${ }^{\text {a* }}$, Anna Stec ${ }^{\mathrm{b}}$, Bhaskar

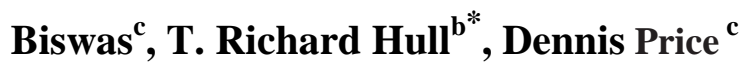 \\ ${ }^{a}$ Center for Degradable and Flame-Retardant Polymeric Materials, College of Chemistry, Sichuan \\ University, Chengdu 610064, China
}

${ }^{b}$ Centre for Fire and Hazards Science, School of Forensic and Investigative Science, University of Central Lancashire, Preston, PR1 2HE, UK

${ }^{c}$ Fire Materials Laboratory, Centre for Material Research and Innovation, University of Bolton, Deane Campus, Bolton BL3 5AB, UK

\begin{abstract}
Polyethylene (PE) was treated with various formulations containing an intumescent fire retardant, which consists of melamine phosphate (MP), pentaerythritol (PER) and ammonium polyphosphate (APP), and one or none of following metal-chelates: CuSAO, CoSAO and NiSAO. The behaviour of this intumescent system can be enhanced significantly by the addition of small amounts $(0.2 \%)$ of metal chelate (CuSAO, CoSAO and NiSAO). The thermal stabilization, burning behaviour and char formation of the fire retardant PE system have been investigated by TGA, LOI, UL-94 test, SEM and cone
\end{abstract}

\footnotetext{
${ }^{*}$ Corresponding author. Tel. \& Fax:+86-28-85410259; E-mail address: yzwang@email.scu.edu.cn(Y. -Z. Wang)

* Corresponding author. Tel: +44 (0)1772 893543; Fax: +44 (0)1772894981 E-mail address:trhull@uclan.ac.uk
} 
calorimetry. All formulations studied provide good fire retardant behaviour, with LOI $\geq 27.4$ and UL-94 V-0 rating. TGA results present more complicated thermal decomposition behaviour after the addition of small amounts $(0.2 \%)$ of metal chelate when compared to that of PE-IFR. Cone calorimetry of PE-IFR-metal chelate (PE-IFR-CuSAO, PE-IFR-CoSAO and PE-IFR-NiSAO) shows a very significant decrease in HRR, PHRR, ML, THR and a very significant improvement of TTI compared to samples without metal chelate. Furthermore, SEM and photographs of the char layer show that the char layer from PE-IFR-metal chelate has a compact and tough char structure compared to the open porous char layer produced by sample without metal chelate.

Key words: chelate; polyethylene; intumescent; fire retardant; burning behaviour 


\section{Introduction}

It is well known that polyethylene (PE) has been widely used in many fields because of its excellent performance, such as low density, low toxicity, excellent electric insulation, mechanical durability, good chemical resistance, ease of processing and moulding, low cost, and so on. Unfortunately, PE burns easily and has poor drip performance. So, there is still a need to improve both fire retardancy and the poor drip performance of PE.

Usually, the common fire retardants for PE can be classified into two kinds: halogen based flame retardants which act in the gas phase; and char forming and intumescent fire retardants (IFR) which incorporate phosphorus or nitrogen in their structures or formulations. However, because of environmental pressure, most halogen based flame retardants are now considered unacceptable. Compared with halogen based flame retardants, intumescent fire retardant (IFR) additives are more promising as an effective and "green" fire retardant for PE based on their excellent intumescent carbonization. Many studies focused on intumescent fire retardant (IFR) have been widely investigated in the fire retardation of polyolefin [1-4]. Generally, they act by a condensed phase mechanism when heating or burning [5-10]. On heating, intumescents form a foamed cellular charred layer on the polymer surface, which acts as a physical barrier and protects the underlying materials from the action of heat and flame. Usually, such formulations contain three ingredients: an acid source, a blowing agent and a carbonization agent. However, the conventional IFR additives also have disadvantages [11-13], such as the higher loading needed in order to obtain the required fire retardant level. Thus, further improvements in the fire retardant efficiency of IFRs are needed.

On the other hand, it is worth noting that some metal chelates can affect the initial stage of polymer degradation [14-16]. In our earlier work, some research on fire retarded PE containing metal chelates has 
been carried out $[17,18]$, meanwhile, reactive extrusion technology was adopted to synthesize a fire retardant (ER), based on the esterification of melamine phosphate (MP) and pentaerythritol (PER) at a particular temperature (shown in Scheme 1). It has been found that ER combined with APP and a small quantity of copper (II)-chelate at pre-selected levels can be an effective IFR for PE yielding good fire retardancy and good anti-dripping behaviour.

In this paper, different metal chelates (Copper (II), Cobalt (II) and Nickel (II)) complexes with salicylaldoxime (SAO) have been investigated. The fire retardant being used in this paper is ER combined with APP. The effect of metal chelates on the thermal stabilization, char formation and burning behaviours of intumescent fire retardant PE has been studied by LOI, UL-94 test, TGA, SEM and cone calorimeter.

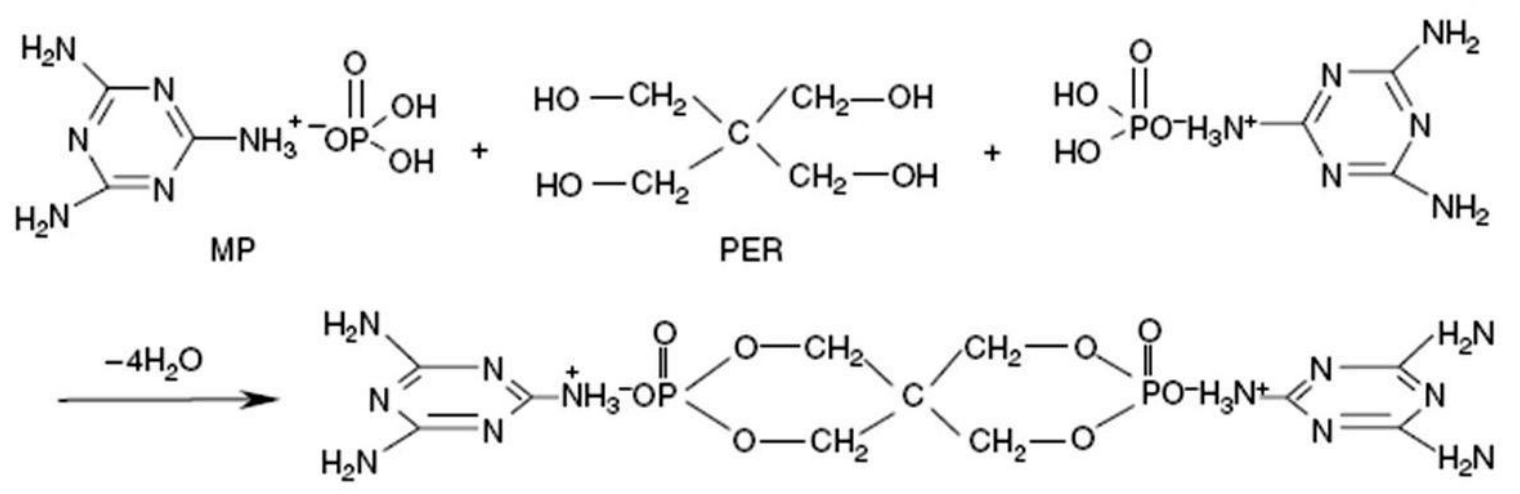

Scheme 1. Reaction of melamine phosphate with pentaerythritol.

\section{Experimental}

\subsection{Materials}

Melamine phosphate (MP), pentaerythritol (PER), sodium hydroxide, methanol and ethanol were provided by Changzheng Chemical Reagent Corp. (Chengdu, China). Salicylaldoxime (SAO), $\mathrm{Cu}\left(\mathrm{CH}_{3} \mathrm{COO}\right)_{2} \cdot \mathrm{H}_{2} \mathrm{O}, \mathrm{Co}\left(\mathrm{CH}_{3} \mathrm{COO}\right)_{2} \cdot 4 \mathrm{H}_{2} \mathrm{O}, \mathrm{Ni}\left(\mathrm{CH}_{3} \mathrm{COO}\right)_{2} \cdot 4 \mathrm{H}_{2} \mathrm{O}$ were supplied by Shanghai First 
Reagent Plant (Shanghai, China). Ammonium polyphosphate (APP) was provided by Changfeng Chemical Corp. (Shifang, China). Reactive extrusion fire retardant (ER) has been prepared in our lab using MP and PER [18]. Commercial low-density polyethylene (LDPE), 112 A, was supplied by Lanzhou Petroleum Chemical Company (Lanzhou, China).

\subsection{Preparation of samples}

\subsubsection{Metal-chelates [19] (CuSAO, CoSAO and NiSAO)}

$\mathrm{M}\left(\mathrm{CH}_{3} \mathrm{COO}\right)_{2} \cdot \mathrm{nH}_{2} \mathrm{O}\left(\mathrm{Cu}\left(\mathrm{CH}_{3} \mathrm{COO}\right)_{2} \cdot \mathrm{H}_{2} \mathrm{O}, \mathrm{Co}\left(\mathrm{CH}_{3} \mathrm{COO}\right)_{2} \cdot 4 \mathrm{H}_{2} \mathrm{O}, \mathrm{Ni}\left(\mathrm{CH}_{3} \mathrm{COO}\right)_{2} \cdot 4 \mathrm{H}_{2} \mathrm{O}\right)(0.01$

mol) was thoroughly milled with $0.02 \mathrm{~mol} \mathrm{SAO}$ at room temperature for $0.5 \mathrm{~h}$, this gave rise to a smell of acetic acid. Then the solid phase reaction was kept $30^{\circ} \mathrm{C}$ for $16 \mathrm{~h}$, and the product, MSAO $(\mathrm{M}=\mathrm{Cu}, \mathrm{Co}, \mathrm{Ni})$ was dried in vacuum $\left(80^{\circ} \mathrm{C}\right)$.

The FTIR spectrum (KBr, pellet) of the metal chelate: $3418-3472 \mathrm{~cm}^{-1}(-\mathrm{OH}), 1520-1580 \mathrm{~cm}^{-1}$ $\left(\mathrm{C}=\mathrm{C}\right.$, phenyl), $430-470 \mathrm{~cm}^{-1}(\mathrm{M}-\mathrm{O}), 1660-1680 \mathrm{~cm}^{-1}(\mathrm{C}=\mathrm{N})$ was determined to confirm the structure of the respective chelate. The structure of the chelates is shown in scheme 2. Analysis of the $\mathrm{C}, \mathrm{H}, \mathrm{N}, \mathrm{O}$ content of metal chelate was carried out on CARLO ERBA1106 elemental analyser. The detailed results were shown as following. CuSAO: $\mathrm{C} \%=49.84$ (calculated 50.07), $\mathrm{H} \%=3.34$ (calculated 3.58 ), $\mathrm{N} \%=8.40$ (calculated 8.35), $\mathrm{O} \%=19.12$ (calculated 19.08); CoSAO: $\mathrm{C} \%=50.72$ (calculated 50.83 ), $\mathrm{H} \%=3.45$ (calculated 3.63), $\mathrm{N} \%=8.52$ (calculated 8.46), $\mathrm{O} \%=19.40$ (calculated 19.34); $\mathrm{NiSAO}: \mathrm{C} \%=50.71$ (calculated 50.80), $\mathrm{H} \%=3.46$ (calculated 3.63), $\mathrm{N} \%=8.51$ (calculated 8.47), $\mathrm{O} \%=19.42$ (calculated 19.35).

This shows good agreement with the proposed formula. 


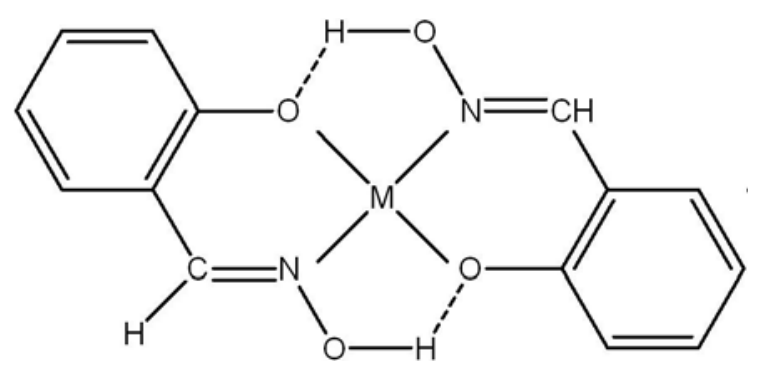

Scheme 2. The structure of MSAO $(\mathrm{M}=\mathrm{Cu}, \mathrm{Co}, \mathrm{Ni})$.

\subsubsection{Fire retardant $P E$ samples}

The total content of IFR ( $\left.\mathrm{W}_{\mathrm{APP}}: \mathrm{W}_{\mathrm{ER}}=2: 1\right)$ in premixed PE was kept at $29.8 \mathrm{wt} \%$. The IFR-PE was prepared by using a counter-rotation twin-screw extruder under the following standard condition: average residence time: $25 \mathrm{~s}$; rotation of screw: $80 \mathrm{rpm}$; mixing temperature: $150^{\circ} \mathrm{C}$. The extrudate was cut into pellets and then extruded again.

All the metal chelates (CuSAO, CoSAO and NiSAO) were introduced into the IFR-PE at a concentration of $0.2 \mathrm{wt} \%$. One sample of $29.8 \mathrm{wt} \%$ IFR-PE had $0.2 \mathrm{wt} \%$ of IFR added as a control sample. All the samples were prepared by mixing all the constituents on a two-roll mill at a roller temperature of $150-160{ }^{\circ} \mathrm{C}$. After mixing, the samples were hot-pressed under $10 \mathrm{MPa}$ for $5 \mathrm{~min}$ at $120^{\circ} \mathrm{C}$ into a sheet of suitable thickness and size as required for the corresponding test standard.

\subsection{Characterization}

\subsubsection{LOI test}

The LOI values were measured on a JF-3 oxygen index meter (Jiangning, China) using sheet dimensions of $130 \times 6.5 \times 3 \mathrm{~mm}^{3}$ according to ASTM D2863-97. 


\subsection{2. $U L-94$ test}

Vertical burning tests were conducted on a vertical burning test instrument (CZF-2-type) (Jiangning, China) using sheet dimensions of $130 \times 13 \times 3 \mathrm{~mm}^{3}$ according to ASTM D3801.

\subsubsection{Thermogravimetric analysis (TGA)}

Thermogravimetric analysis (TGA) was performed on a DuPont 1090B thermogravimetric analyzer at a heating rate of $10{ }^{\circ} \mathrm{C} / \mathrm{min} .7 \mathrm{mg}$ of samples were examined under flowing air $(80 \mathrm{ml} / \mathrm{min})$ over a temperature range from room temperature to $600{ }^{\circ} \mathrm{C}$. Each determination was carried out in duplicate.

\subsubsection{Scanning electron microscopy (SEM)}

A HITACHI MODEL: S-450 scanning electronic microscopy (SEM) was used to investigate the outer surface of residues of PE-IFR and PE-IFR-CuSAO. The residue samples for SEM were obtained after two samples were burnt directly in air. The micrographs of the residual char samples were recorded after surface treatment using a sputtered deposition of gold.

\subsubsection{Cone calorimeter}

The cone calorimeter tests were carried out following the procedures indicated in the ISO 5660 standard with a FTT cone calorimeter. Square specimens $(100 \times 100 \times 6 \mathrm{~mm})$ were irradiated at a heat flux of $25 \mathrm{~kW} / \mathrm{m}^{2}$. 


\section{Results and discussion}

\subsection{LOI and UL-94 test}

As is well known, LOI measurement and UL-94 test are widely used to evaluate the fire retardant properties of materials. In this paper, all the samples have been tested using LOI and UL-94 measurement according to ASTM D2863-97 and ASTM D380, respectively. The LOI values, UL-94 rating and dripping behaviour of all the specimens is shown in table 1.

\begin{tabular}{|c|c|c|c|c|c|c|}
\hline \multicolumn{4}{|c|}{ Composition of Fire-Retardant PE } & \multirow{2}{*}{$\begin{array}{c}\text { LOI values } \\
\quad \%\end{array}$} & \multirow{2}{*}{$\begin{array}{l}\text { UL-94 } \\
\text { Rating }\end{array}$} & \multirow[t]{2}{*}{ Drip } \\
\hline PE(wt\%) & $\operatorname{IFR}(w t \%)$ & Metal chelate & Conc (wt \%) & & & \\
\hline 100 & 0 & -- & -- & 18 & No Rating & Heavily \\
\hline 70 & 30 & -- & -- & 27 & $\mathrm{~V}-0$ & No \\
\hline 70 & 29.8 & CuSAO & 0.2 & 29 & $\mathrm{~V}-0$ & No \\
\hline 70 & 29.8 & CoSAO & 0.2 & 28 & $\mathrm{~V}-0$ & No \\
\hline 70 & 29.8 & NiSAO & 0.2 & 28 & $\mathrm{~V}-0$ & No \\
\hline
\end{tabular}

Table 1 Effect of the composition of IFR-metal chelate on fire retardancy of PE with $30 \mathrm{wt} \%$ total loading level of additives.

From table 1, it can be observed that the LOI values of PE increased from 18 to 27 when $30 \mathrm{wt} \%$ IFR is added to PE, simultaneously, the UL-94 reached V-0 rating showing both reduced flame spread and no dripping during burning. More interestingly, it was observed that the LOI values increased modestly from 27 to 29,28 and 28 respectively with the addition to the fire retardant PE of only $0.2 \mathrm{wt} \%$ metal chelate (CuSAO, CoSAO and NiSAO), while maintaining $30 \mathrm{wt} \%$ loading of fire retardant. 


\subsection{Thermogravimetric analysis (TGA)}

In order to understand the effects of metal chelate (CuSAO, CoSAO and NiSAO) for intumescent fire retardant PE, we compared the thermal degradation behaviour and the mass of residue obtained for the various samples. TGA testing of PE, PE-IFR, PE-IFR-CuSAO, PE-IFR-CoSAO and PE-IFR-NiSAO was carried out in air at a heating rate $10^{\circ} \mathrm{C} / \mathrm{min}$. TG and DTG curves are presented in figure 1 and figure 2, respectively, and some detailed data shown in table 2.

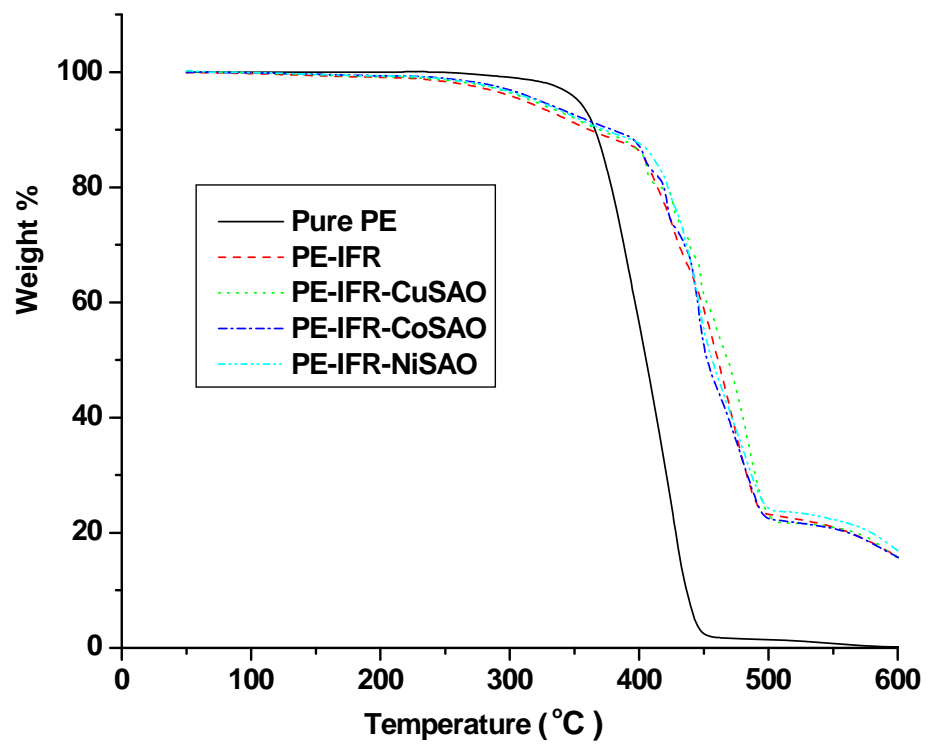

Fig. 1. TG curves of PE, PE-IFR, and PE-IFR-metal chelate systems in air. 


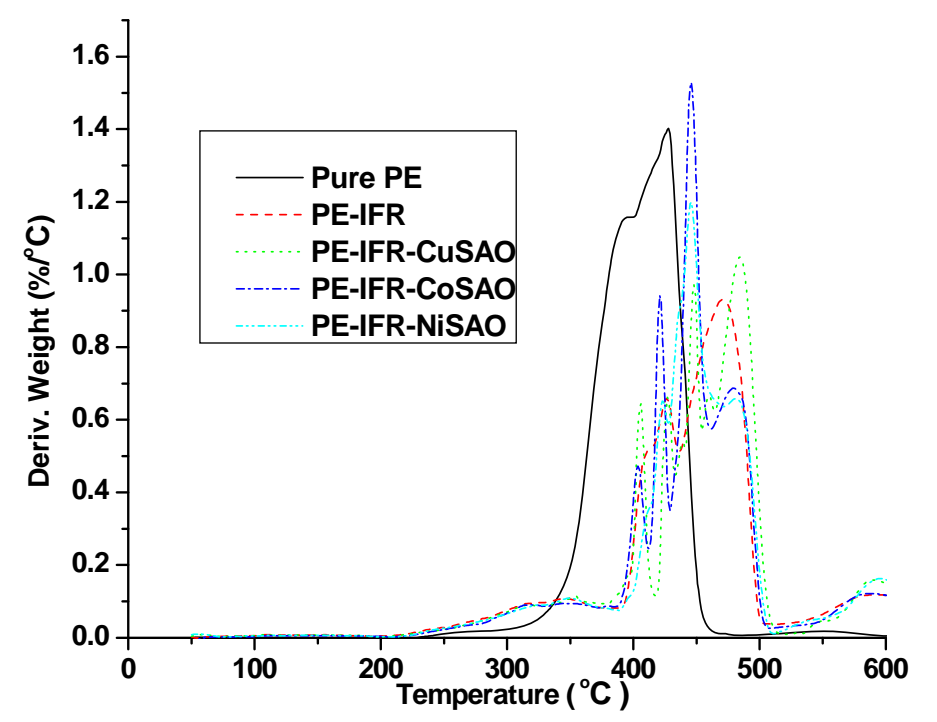

Fig. 2. DTG curves of PE, PE-IFR, and PE-IFR-metal chelate systems in air.

It is as expected that PE-IFR and PE-IFR-Metal chelates (CuSAO, CoSAO and NiSAO) increase the residue yield significantly by comparison with pure PE. However, it is more interesting to look at the difference in the thermal degradation behaviour of intumescent fire retardant PE based upon the addition of only 0.2 wt $\%$ metal chelates. It can be observed from table 2 that $\mathrm{T}_{\text {onset }}$ of PE-IFR-metal chelate (CuSAO, CoSAO and NiSAO) are modestly enhanced compared with that of PE-IFR, while comparison of $\mathrm{T}_{\max }$ and $\mathrm{T}_{50 \%}$ shows no clear pattern. Even so, it is worth noticing that the $\mathrm{T}_{\max }$ and $\mathrm{T}_{50 \%}$ of all samples are between 400 and $500{ }^{\circ} \mathrm{C}$. Meanwhile from figure 2, it can be observed that DTG curves of fire retardants containing metal chelates are significantly more complex than that without metal chelate over the temperature range. For PE-IFR, DTG curve shows only two peaks between $400-500{ }^{\circ} \mathrm{C}$, while over the same temperature range, the number of peaks in DTG curves is four, four and three for PE-IFR-CuSAO, PE-IFR-CoSAO, PE-IFR-NiSAO, respectively. This suggests that when subjected to heating or burning the addition of the metal chelate correspondingly complicates the reactions taking place. When the residue yields at $600{ }^{\circ} \mathrm{C}$ are compared, it is found that although PE-IFR, PE-IFR-CuSAO 
and PE-IFR-CoSAO are similar at about $15.8 \%$, the yield from PE-IFR-NiSAO is somewhat greater at about $16.9 \%$. This implies a greater degree of carbonisation under TGA condition. The TGA data combined with the LOI data suggest that the metal chelate can enhance the intumescent fire retardant in PE.

\begin{tabular}{ccccc}
\hline sample & $\mathbf{T}_{\mathbf{\text { onset }}}{ }^{\mathbf{a}}\left({ }^{\circ} \mathbf{C}\right)$ & $\mathbf{T}_{\mathbf{5 0 \%}}{ }^{\mathbf{b}}\left({ }^{\circ} \mathbf{C}\right)$ & $\mathbf{T}_{\mathbf{m a x}}{ }^{\mathbf{C}}\left({ }^{\circ} \mathbf{C}\right)$ & $\mathbf{Y}^{\mathbf{d}} \mathbf{( \% )}$ \\
\hline PE & 353 & 405 & 428 & 0.1 \\
PE-IFR & 311 & 461 & 471 & 15.9 \\
PE-IFR-CuSAO & 317 & 469 & 484 & 15.7 \\
PE-IFR-CoSAO & 323 & 453 & 446 & 15.7 \\
PE-IFR-NiSAO & 321 & 457 & 446 & 16.9 \\
\hline
\end{tabular}

a): $\mathrm{T}_{\text {onset }}$ the on-set degradation temperature (temperature at $5 \%$ weight loss). b): $\mathrm{T}_{50 \%}$ the half degradation temperature (temperature at the half amount of the initial weight loss). c): $\mathrm{T}_{\max }$ the maximum-rate degradation temperature. d): $\mathrm{Y}$ the residue at $600{ }^{\circ} \mathrm{C}$

Table 2 Calculated results from TG curves of various samples (heating rate: $10^{\circ} \mathrm{C} / \mathrm{min}$ ).

\subsection{Scanning electron microscopy (SEM)}

In order to investigate the relationship between the structure of intumescent chars formed and the fire-resistant properties of the polymer compositions, the char residues left after burning in air were examined by SEM. Figure 3 presents the SEM micrographs of intumescent chars obtained from PE-IFR and PE-IFR-CuSAO at different magnifications. Significant differences between the SEM micrographs can be observed. From figure 3 (a) and (c), the char layer from PE-IFR shows a open porous char structure. Whereas the char structure obtained from PE-IFR-CuSAO shown in figure 3 (b) and (d) is more compact and appears to have a composed of platelets structure, which would be a more effective harrier. Generally the speed of formation of the protective char layer and the performance of char layer are highly dependant upon factors such as fire retardant, fire temperature and the viscosity of the molten polymer. Comparing the char layers of PE-IFR and PE-IFR-CuSAO, suggests that the addition of small amounts of 
CuSAO induces processes, such as crosslinking or dehydration, and changes the morphology of the carbonization compounds formed during burning, so that a char layer with improved performance is formed. Furthermore, it is likely that this is related to the increase in the viscosity of the molten mixture resulting from early catalytic oxidation. This is in accord with the results from TG analysis. It is well known that the intumescent char structure acts as an insulating barrier, can prevent heat and fuel transfer between the flame zone and the underlying substrate, and thus protect the substrate from heat and fire. It is apparent that the fire-resistant performance of these materials is directly connected with the quality of this barrier. SEM analysis demonstrates that the intumescent fire retardant with small amounts of metal chelate produces a more closed char and as a consequence has potentially better fire-retardant properties than that without metal chelate. This is in line with the LOI results.

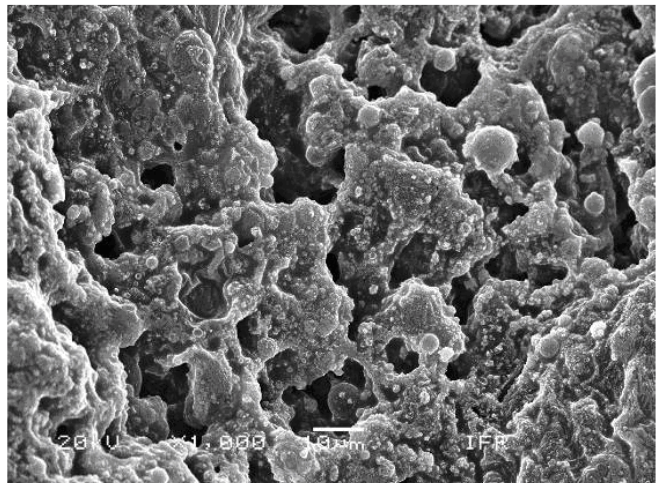

(a) PE-IFR (1000x)

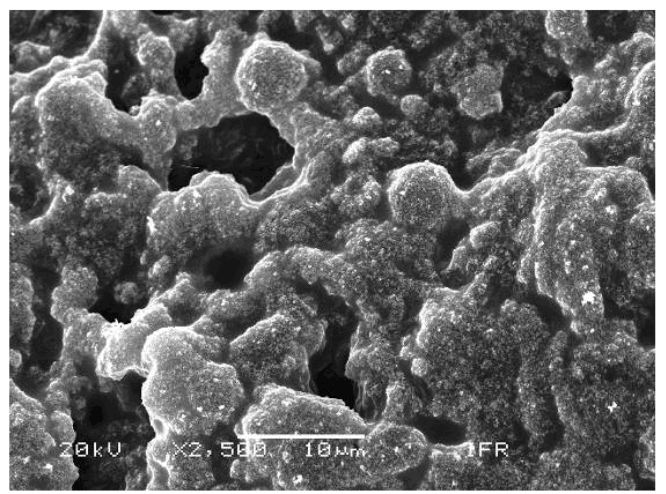

(C) PE-IFR (2500x)

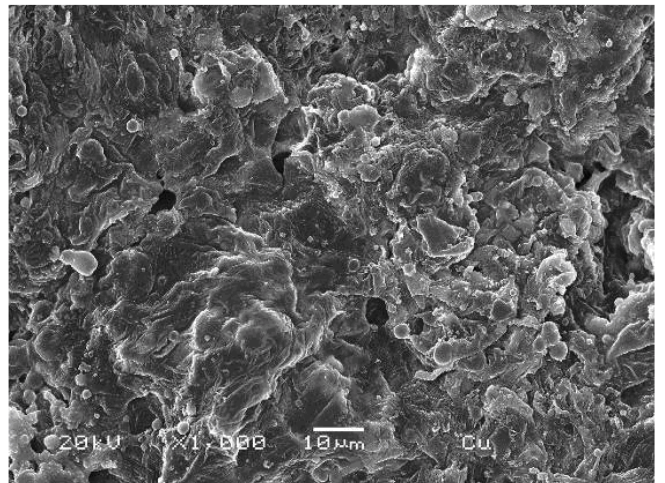

(b) PE-IFR-CuSAO (1000x)

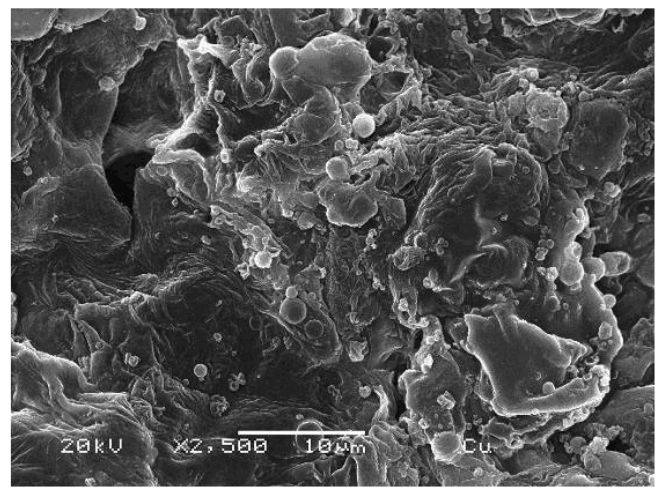

(d) PE-IFR-CUSAO (2500x)

Fig. 3. SEM images of char layer from PE-IFR and PE-IFR-CuSAO after burning in air: (a) PE-IFR

(1000×); (b) PE-IFR-CuSAO (1000×); (c) PE-IFR (2500×) and (d) PE-IFR-CuSAO (2500×). 


\subsection{Cone calorimeter results}

The cone calorimeter is a performance based bench scale fire testing apparatus and provides a wealth of information on combustion behaviour [20]. Some cone calorimeter results have been found to correlate well with those obtained from large scale fire tests, so that it can be used to predict the behaviour of materials in a real fire [21]. Furthermore, it provides comprehensive insight into fire risk via parameters such as heat release rate (HRR), total heat release (THR) and time to ignition (TTI). Thus the cone calorimeter is a useful tool for the evaluation of fire retardant materials. Figure 4 shows curves of the heat release rate (HRR) of pure PE, PE-IFR, PE-IFR-CuSAO, PE-IFR-CoSAO and PE-IFR-NiSAO. Pure PE burns very rapidly after ignition and a sharp HRR peak appears with a peak heat release rate (PHRR) of $734 \mathrm{~kW} / \mathrm{m}^{2}$. Of more interest is the difference in HRR between materials with and without metal chelate. The PHRR of PE-IFR is $278 \mathrm{~kW} / \mathrm{m}^{2}$, while PE-IFR-CuSAO, PE-IFR-CoSAO and PE-IFR-NiSAO show a lower burning rate with the peak reduced to $166,169,147 \mathrm{~kW} / \mathrm{m}^{2}$ respectively, only $60 \%, 61 \%$ and $53 \%$ of that of PE-IFR and only $23 \%, 23 \%$ and $20 \%$ of that of pure PE, the detailed data is shown in table 3. The results suggest that the addition of only small amounts of metal chelate shows a considerable effect on the IFR system. 


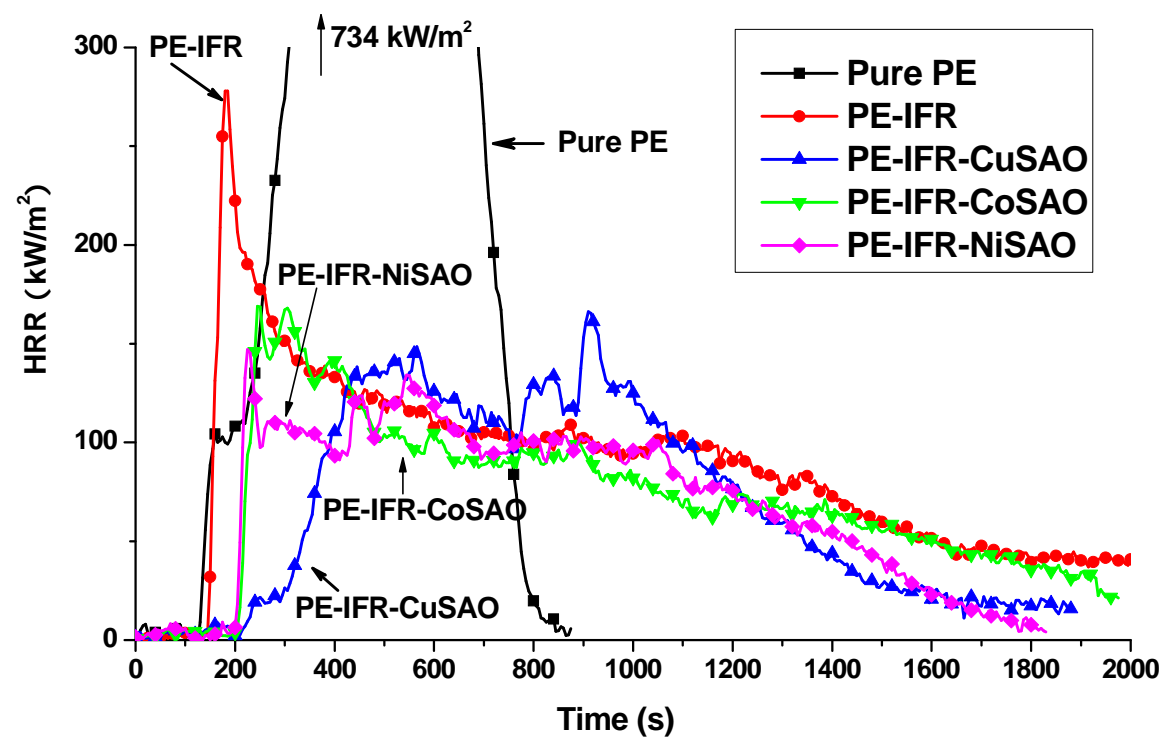

Fig. 4. Comparison of heat release rate curves of studied samples.

\begin{tabular}{cccccc}
\hline Sample & TTI (s) & $\begin{array}{c}\text { Av-RHR } \\
\left(\mathrm{kW} / \mathrm{m}^{2}\right)\end{array}$ & $\begin{array}{c}\text { PHRR } \\
\left(\mathrm{kW} / \mathrm{m}^{2}\right)\end{array}$ & $\begin{array}{c}\text { THR } \\
\left(\mathrm{MJ} / \mathrm{m}^{2}\right)\end{array}$ & $\begin{array}{c}\text { Residual } \\
\text { Mass } \\
(\%)\end{array}$ \\
\hline PE & 132 & 352 & 806 & 239 & 0.9 \\
PE-IFR & 166 & 71 & 278 & 173 & 31.6 \\
PE-IFR-CuSAO & 268 & 67 & 166 & 127 & 46.2 \\
PE-IFR-CoSAO & 218 & 70 & 169 & 137 & 46.0 \\
PE-IFR-NiSAO & 217 & 67 & 147 & 125 & 46.8 \\
\hline
\end{tabular}

TTI: time to ignition

Av: average

Residual Mass\%: mass percentage left when testing finished

Table 3. Combustion parameters obtained from cone calorimeter.

However, the most notable difference in the experiments using the cone calorimeter comes from time to ignition (TTI). The TTI of pure PE and PE-IFR are $132 \mathrm{~s}$ and $166 \mathrm{~s}$, respectively. However, the TTI of PE-IFR-CuSAO, PE-IFR-CoSAO and PE-IFR-NiSAO are 268 s, $218 \mathrm{~s}$ and 217 s, respectively, which is 
much longer than those of pure PE and PE-IFR. We believe that this result from improvements in the performance of the protective char layer formed prior to ignition. It indicates that an ignition resistant protective char layer was formed after addition of small quantities of metal chelate. This is in accord with the results from SEM. The samples studied showed considerable differences in the Total Heat Release (THR/Time) curves presented in figure 5.

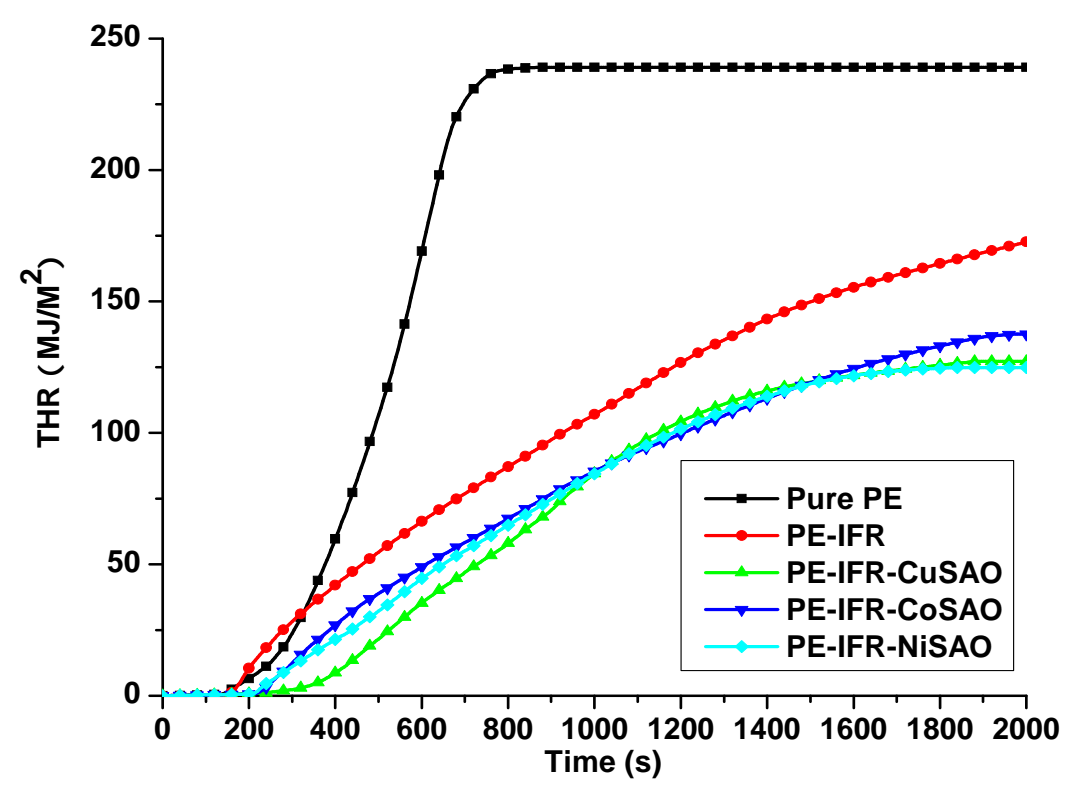

Fig. 5. Comparison of total release rate curves of studied samples.

At the end of burning, pure PE has released a total heat of $239 \mathrm{MJ} / \mathrm{m}^{2}$, the PE-IFR has released is 173 $\mathrm{MJ} / \mathrm{m}^{2}$, whereas PEIFR-Metal chelates, only released $127 \mathrm{MJ} / \mathrm{m}^{2}, 137 \mathrm{MJ} / \mathrm{m}^{2}$, and $125 \mathrm{MJ} / \mathrm{m}^{2}$, respectively, for PE-IFR-CuSAO, PE-IFR-CoSA and PE-IFR-NiSAO. This lower THR value indicates that a part of the PE-IFR-Metal chelate has not completely combusted, probably undergoing a carbonization process. This is in accord with the mass loss, and the formation of residues, which are also presented in figure 6,7 , respectively. 


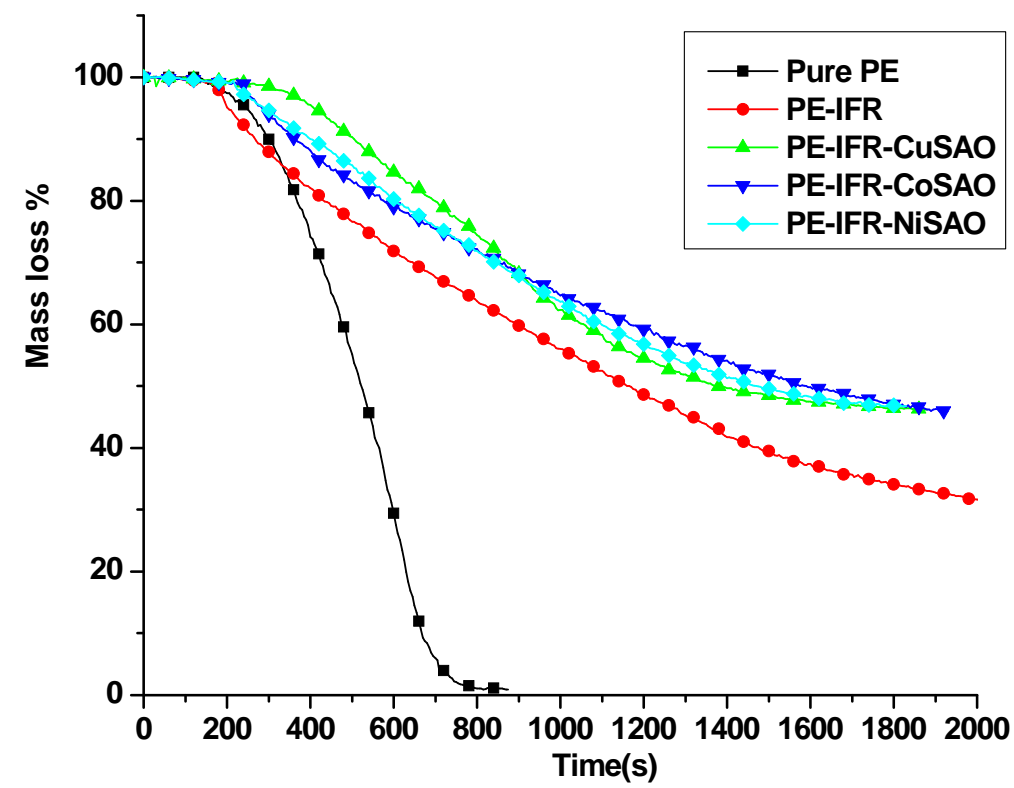

Fig. 6. Comparison of mass loss curves of studied samples.

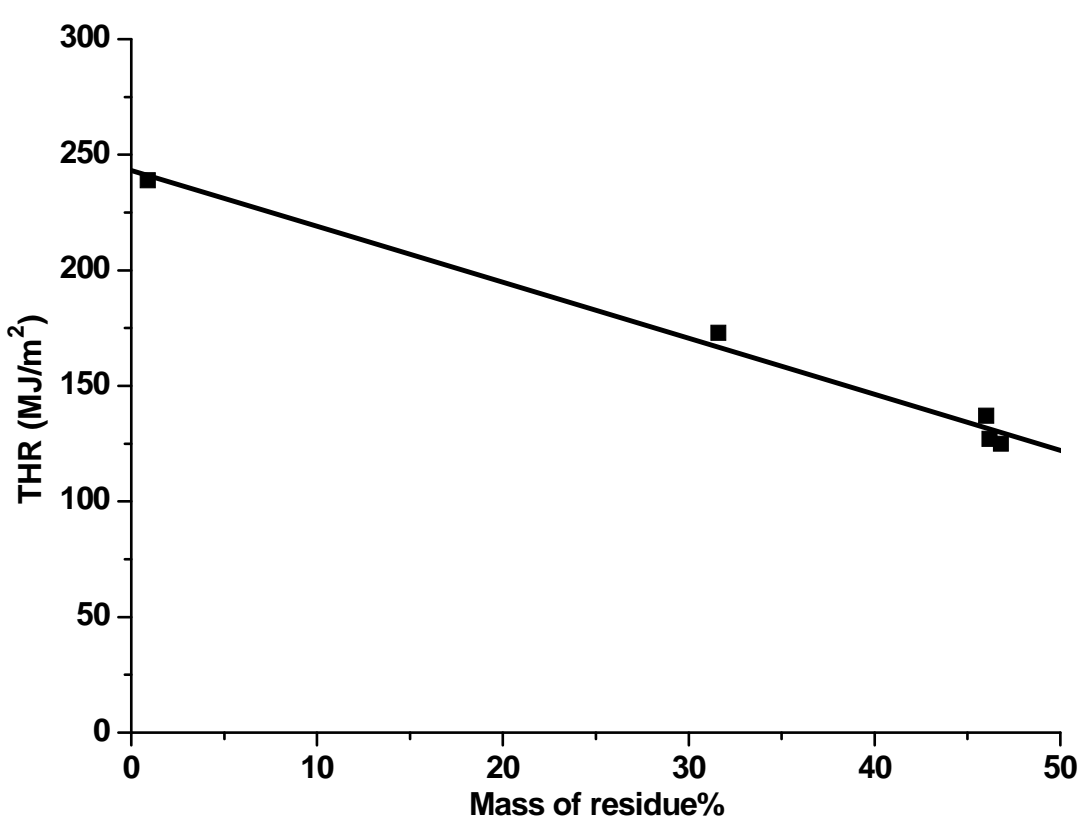

Fig. 7. Relationship of residual mass and THR of studied samples.

Figure 6 shows weight loss as a function of combustion time for pure PE and PE-IFR in comparison with PE-IFR-Metal chelates (PE-IFR-CuSAO, PE-IFR-CoSAO and PE-IFR-NiSAO). It can be seen that the PE-IFR-Metal chelates present a significantly lower mass loss (ML). Meanwhile, the relationship of 
residual mass and THR of studied samples has presented in figure 7.

The figure 7 shows that the mass of residue is in proportion to the THR. This confirms the proposed mechanism of increased char yield as a means of reducing the heat release, for the early flaming condition of $25 \mathrm{~kW} / \mathrm{m}^{2}$. However, the availability of cone plaques for this work was limited. When tested at 35 $\mathrm{kW} / \mathrm{m}^{2}$ and $50 \mathrm{~kW} / \mathrm{m}^{2}$, the difference in THR were not observed between the metal chelate sample and the PE-IFR. However, at $35 \mathrm{~kW} / \mathrm{m}^{2}$, the CuSAO sample showed a significantly greater ignition delay time than the other samples.

Observation of the burning process and the residues remaining after the cone calorimeter test showed pure PE burns rapidly after ignition with almost no residue left. By comparison PE-IFR and PE-IFR-Metal chelate all show an obvious intumescent char layer. However, there are significant differences in the burning behaviour and the performance both the inner and outer char materials. In the initial stages, the flaming of PE-IFR is more intense than that of PE-IFR-Metal chelate, particularly the PE-IFR-CuSAO and PE-IFR-NiSAO. The latter show candle-like burning from fuel evolved through small hole in the protective layer. The PE-IFR-Metal chelate residues showed an obviously metallic lustre on their surface, suggesting more complete graphitization. The residue on the PE-IFR surface was quite loose and friable by comparison with the residues from the PE-IFR-Metal chelates. The tougher outer layer produce by the metal chelate IFR systems would prevent the effective transfer of gas and volatiles through the surface, so preventing burning. The presence of the metal chelate seems to inhibit significantly the decomposition process. It was observed that there were significant differences between the materials with respect to the inner layer of the residues. It was observed that the residue from PE-IFR-Metal chelate had a lower liquid layer (probably olefinic) arising from the incomplete combustion of the inner layer. The presence of this residence was not observed with the PE-IFR system. After cooling 
down, the thickness of the protective layers was measured using callipers. The average thickness of the layers for different samples is shown in table 4. It is known that the thicker the layer, the more difficult transfer of gas and volatile will be, and assuming comparable densities, the greater the amount of fuel retained in the char. From the results of table 4, it can be observed that the residue layers from the system containing metal chelate are thicker than from PE-IFR. Thus they can offer a more effective protection and prevent the transfer of the gas, volatiles and heat. The result is in accord with the results from SEM. So it is concluded that there is an important role for low concentration of metal chelate in the improvement of the performance of a char layer during burning.

\begin{tabular}{cccccc}
\hline sample & PE & PE-IFR & $\begin{array}{c}\text { PE-IFR- } \\
\text { CuSAO }\end{array}$ & $\begin{array}{c}\text { PE-IFR-Co } \\
\text { SAO }\end{array}$ & $\begin{array}{c}\text { PE-IFR- } \\
\text { NiSAO }\end{array}$ \\
\hline $\begin{array}{c}\text { Thickness of } \\
\text { Residue Layer } \\
(\mathrm{mm})\end{array}$ & 0 & 2.0 & 2.6 & 2.3 & 2.5 \\
\hline
\end{tabular}

Table 4. The average thickness of residues layers for samples.

\section{Conclusions}

In this study, PE was treated with various formulations containing an IFR and different metal-chelates (CuSAO, CoSAO and NiSAO). The effect of very small amounts of the metal chelates on the thermal stabilization and burning behaviour is apparent, showing inhibition of thermal decomposition. By cone calorimetry, PE-IFR-metal chelate (PE-IFR-CuSAO, PE-IFR-CoSAO and PE-IFR-NiSAO) shows very significant decrease in HRR, PHRR, ML, THR and very considerable improvement in TTI compared to materials without metal chelate. Furthermore, it is significant to observe from SEM and observation of the char layer that PE-IFR-metal chelate system produces a compact and tough char 
structure compared to a loose porous char layer formed without metal chelate. The reason may be related to the catalysis (such as crosslinking or dehydration) leading to enhanced graphitization, but this is not yet clear. Further work will be needed to study and understand the fire retardant mechanism and obtain more direct evidence.

\section{Acknowledgements}

This work was supported by the National Science Fund for Distinguished Young Scholars (50525309). One of us (De-Yi Wang) would like to thank the University of Bolton for the provision of a visiting fellowship and the support of Innovation Funds of Students of Sichuan University.

\section{References}

[1] Bras ML, Bugajny M, Lefebvre JM, Bourbigot S. Use of polyurethanes as char-forming agents in polypropylene intumescent formulations. Polym Int 2000; 49: 1115.

[2] Zhu WM, Weil ED, Mukhopadhyay S. Intumescent flame-retardant system of phosphates and $5,5,5^{*}, 5^{\prime \prime}, 5^{\prime \prime}, 5^{\prime \prime}$-hexamethyltris (1,3,2-dioxaphosphorinanemethan)amine $2,2^{*}, 2^{\prime \prime}$ trioxide for polyolefins. J Appl Polym Sci 1996; 62: 2267.

[3] Ravadits I, Toth A, Marosi G, Ma'rton A, Sze'p A. Organosilicon surface layer on polyolefins to achieve improved flame retardancy through an oxygen barrier effect. Polym Degrad Stab 2001; 74: 419.

[4] Xie RC, Qu BJ. Expandable graphite systems for halogen-free flame-retarding of polyolefins. I. Flammability characterization and synergistic effect. J Appl Polym Sci 2001; 80: 1181. 
[5] Kandola BK, Horrocks AR. Complex char formation in flame-retarded fibre-intumescent combinations_-II. Thermal analytical studies. Polym Degrad Stab 1996; 54: 289.

[6] Camino G, Intumescent Fire-retardant Systems. Polym Degrad Stab 1989; 23: 359.

[7] Horacek H, Pieh S. The importance of intumescent systems for fire protection of plastic materials. Polym Int 2000; 49: 1106.

[8] Horacek H, Grabner R, Advantages of flame retardants based on nitrogen compounds. Polym Degrad Stab 1996; 54: 205.

[9] Levchik SV, Costa L, Inhibiting action of polymeric hindered amines on the oxidation of 1,4-polybutadiene by singlet oxygen. Polym Degrad Stab 1992; 36: 229.

[10] Chiu SH, Wang W K. The dynamic flammability and toxicity of magnesium hydroxide filled intumescent fire retardant polypropylene. J Appl Polym Sci 1998; 67: 989.

[11] Ou YX. Applied Flame-retarding Technology. Beijing: Chemical Industrial Press; 2002.

[12] Chiang WY, Hu HH. Phosphate-containing flame-retardant polymers with good compatibility to polypropylene. II. Effect of the flame-retardant polymers on polypropylene. J Appl Polym Sci 2001; 82; 2399.

[13] Ma ZL, Zhao M, Hu HF, Ding HT, Zhang J. Compatibilization of intumescent flame retardant/polypropylene composites based on $\mathbf{\alpha}$-methacrylic acid grafted polypropylene. J Appl Polym Sci 2002; 83: 3128.

[14] Ranaweera RPR, Scott G. Mechanisms of antioxidant action: Antioxidant behaviour of nickel complex u.v. stabilisers. Eur Polym J 1976; 12: 825.

[15] Holcik J, Kosik M, Benbow AW, Cullis CF. The oxidative thermal degradation of polypropylene and the influence of transition metal chelates. Eur Polym J 1978; 14 : 
769.

[16] Laver HS, Scott G. Development in Polymer Stabilisation. London: Vol. 1, Applied Science; 1979.

[17] Xie F, Wang YZ, Yang B, Liu Y, A Novel Intumescent Flame-Retardant Polyethylene System. Macro Mater Eng 2006; 291: 247.

[18] Wang DY, Liu Y, Wang YZ, Artiles CP, Hull TR, Price D, Fire retardancy of a reactively extruded intumescent flame retardant polyethylene system enhanced by metal chelates, Polym. Degrad. Stab. 2007, 92, 1592-1598.

[19] Lin JJ, Zheng LM, Fu Y, Studies on Solid State Reactions of Coordination Chemistry (LXV)-The Solid State Reactions of Copper Acetate Monohydrate with Schiff Bases at Room Temperature. Chemical Research in Chinese Universities. 1994; 15: 1110.

[20] Price D, Liu Y, Hull TR, Milnes GJ, Kandola BK, Horrocks AR. Flame retardance of poly(methyl methacrylate) modified with phosphorus-containing compounds. Polym Degrad Stab 2002; 77: 227.

[21] Babrauskas V. SFPE Technology Report 84-10.Boston: Society of Fire Protection Engineers; 1984. 